Super-Poissonian Shot Noise in Tunneling through Coupled Self-Assembled InAs Quantum Dots

P. Barthold, F. Hohls, N. Maire, K. Pierz, and R. J. Haug

Citation: AIP Conference Proceedings 893, 815 (2007); doi: 10.1063/1.2730140

View online: https://doi.org/10.1063/1.2730140

View Table of Contents: http://aip.scitation.org/toc/apc/893/1

Published by the American Institute of Physics

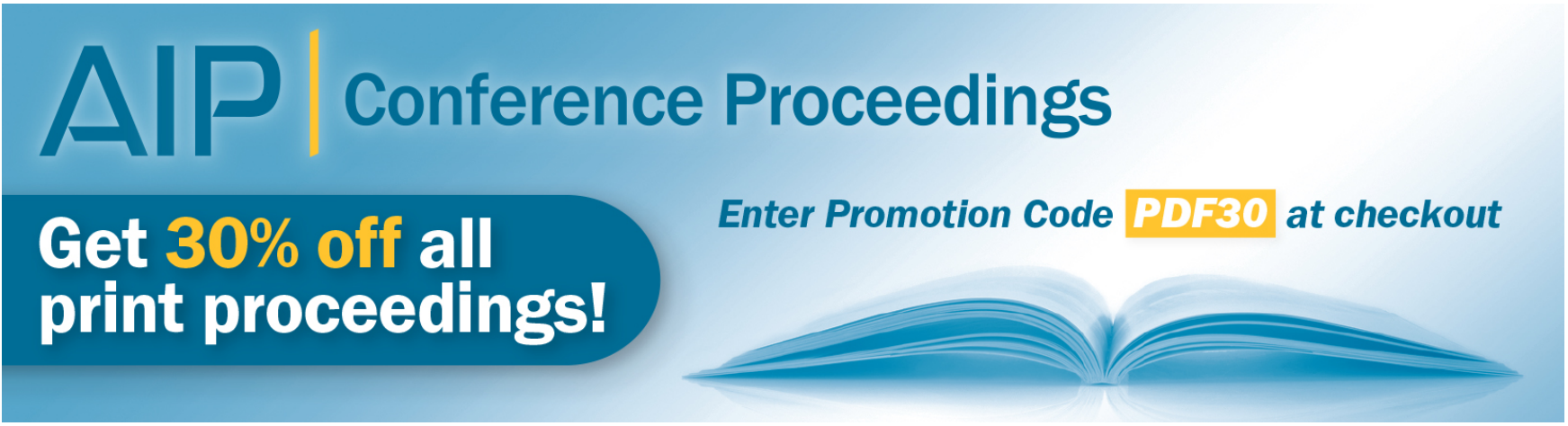




\title{
Super-Poissonian Shot Noise in Tunneling through Coupled Self-Assembled InAs Quantum Dots
}

\author{
P. Barthold*, F. Hohls ${ }^{*, \dagger}$, N. Maire*, K. Pierz ${ }^{* *}$ and R. J. Haug* \\ ${ }^{*}$ Institut für Festkörperphysik, Leibniz Universität Hannover, Appelstrasse 2, 30167 Hannover, Germany \\ ${ }^{\dagger}$ Cavendish Laboratory, University of Cambridge, Madingley Road, Cambridge CB3 OHE, UK \\ ${ }^{* *}$ Physikalisch-Technische Bundesanstalt, Bundesallee 100, 38116 Braunschweig, Germany
}

\begin{abstract}
We apply an external bias voltage to vertically coupled self-assembled InAs quantum dots. We observe pronounced peaks in the I-V characteristic due to resonant transport through a stack of coupled quantum dots. We investigate the noise properties at these peaks and we find an astonishing enhancement of the shot noise at low temperatures with a distinct doublepeak structure.
\end{abstract}

Keywords: enhanced shot noise, coupled quantum dots PACS: $73.63 . \mathrm{Kv}, 72.70 .+\mathrm{m}, 73.40 . \mathrm{Gk}$

The so-called shot noise was found in 1918 by Walter Schottky in vacuum tubes [1]. The current fluctuates around its average value due to the discreteness of the charge. The average shot noise $S$ is proportional to the average current $I: S=2 e I$, where $e$ is the elementary charge. It has been shown that investigating the shot noise properties on tunneling devices provides more information than the DC current alone $[2,3,4,5]$.

The active part of the device consists of a GaAs-AlAs heterostructure with embedded InAs QDs (for further details of the sample see $[6,7]$ ). The quantum dots are realized by two layers of InAs sandwiched between $5 \mathrm{~nm}$ AlAs barriers. Due to Stranski-Krastanov-growth the InAs quantum dots are randomly formed and vertically aligned [8]. By applying an external bias voltage $V_{S D}$ we drive a current through the device which is amplified by a low-noise current amplifier. Parallel to the measured DC current the AC part is analyzed by a spectrum analyzer using Fast Fourier Transformation (FFT) technique. In Fig. 1 the current-voltage (I-V) characteristic for $T=1.4 \mathrm{~K}$ of the investigated sample is presented. Different well defined peaks are obvious. The inset in Fig. 1 helps to understand these peaks in the I-V characteristic. The sketch shows the energy band structure of the sample for an external bias voltage $V_{S D}$ applied to the device. Without any external bias voltage the ground states of the QDs are above the Fermi energy $E_{F}$ of the emitter and collector, therefore no current flows at $V_{S D}=0 \mathrm{mV}$. At a certain voltage $V_{P e a k}$, e.g. $V_{S D}=-186.8 \mathrm{mV}$, both energy levels in the QDs are in resonance and electrons are able to tunnel from the emitter into the QDs and into unoccupied states in the collector. Due to this sequential tunneling a peak in the I-V characteristic occurs [6, 9].

In Fig. 2 three typical current noise spectra are shown

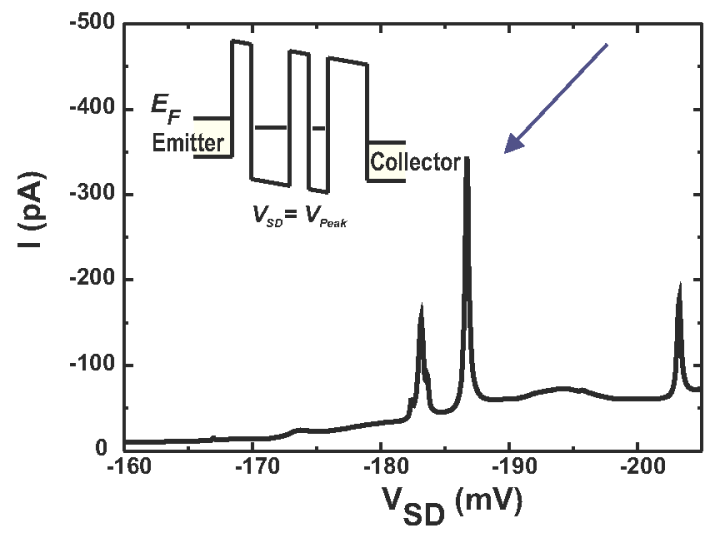

FIGURE 1. Current-Voltage characteristic of the device at $T=1.4 \mathrm{~K}$. The marked peak at $V_{S D}=-186.8 \mathrm{mV}$ will be discussed further in this paper. The inset shows the energy band structure under a bias voltage $\left(V_{S D}=V_{P e a k}\right)$.

for different bias voltages in the range of the marked current peak at $T=1.4 \mathrm{~K}$. At very low frequencies the spectra show a $1 / \mathrm{f}-$ behavior. We fit the function $A / f+S_{0}$ to the spectra to obtain information about the amplitude of the shot noise $S_{0}$. By comparing $S_{0}$ to the averaged noise spectra between $5 \mathrm{kHz}$ and $12.8 \mathrm{kHz}$ we find that $S_{0}$ provides reliable information. As can be seen in Fig. 2 the $1 / f$ part is more than one order of magnitude smaller than the shot noise amplitude in the interesting range between 5 and $12.8 \mathrm{kHz}$.

The so-called Fano factor $\alpha$ compares the fullPoissonian shot noise $S$, that is expected for a single tunneling barrier, with the measured shot noise $S_{0}$ : $\alpha:=S_{0} / S=S_{0} /(2 e I)$. In Fig. 3(a) a blow up of the current peak at $V_{S D}=-186.8 \mathrm{mV}$ is shown for different temperatures $(T=1.4 \mathrm{~K}$ and $T=2.7 \mathrm{~K})$. The Fano 


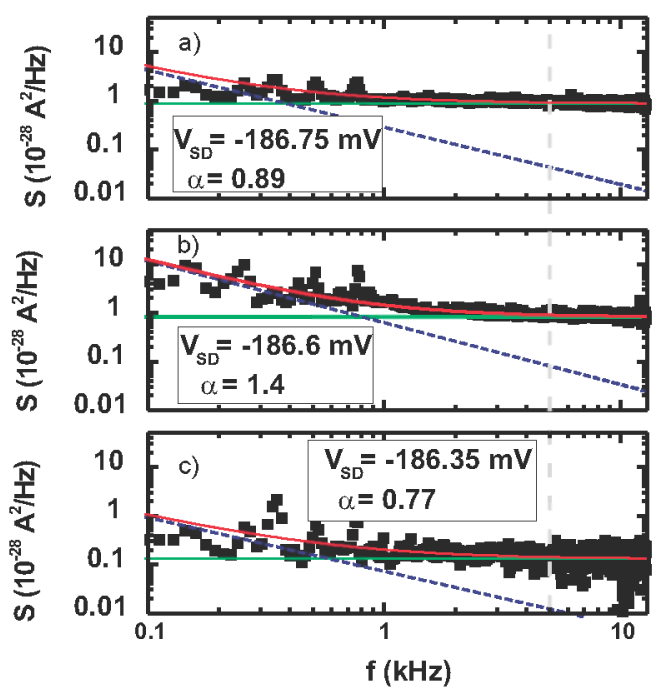

FIGURE 2. Typical noise spectra of the sample for different bias voltages at $T=1.4 \mathrm{~K}$. The squares demonstrate the measured noise spectra, the fitting function $A / f+S_{0}$ is represented by the curve, the horizontal line demonstrates $S_{0}$, the dashed line shows the $1 / f$, the vertical dashed line indicates $5 \mathrm{kHz}$.

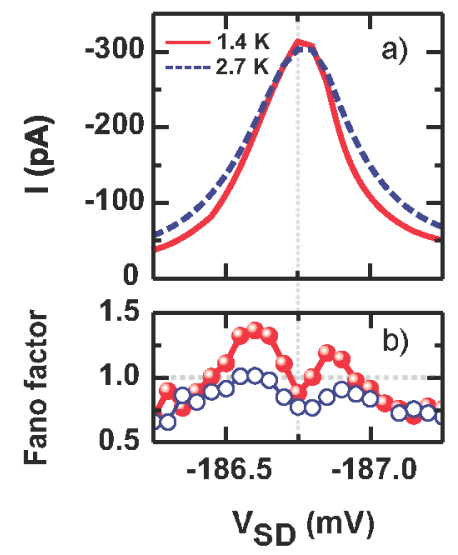

FIGURE 3. (a) Blow up of the marked current peak in Fig. 1 measured at $T=1.4 \mathrm{~K}$ and $T=2.7 \mathrm{~K}$ (dashed line). (b) The filled circles demonstrate the Fano factor $\alpha$ at $T=1.4 \mathrm{~K}$ and the open symbols show $\alpha$ at $T=2.7 \mathrm{~K}$.

factor in this range is shown in Fig. 3(b). We observe a pronounced double-peak behavior in the Fano factor: the shot noise is enhanced on both sides of the current peak and is reduced on top of the current peak. The current changes only slightly with rising temperature whereas the shot noise shows a strong temperature dependence. Theoretical models that take only one stack of coupled quantum dots without any further coupling mechanisms into account like Ref. [10] predict a Fano factor below 1 and therefore a suppressed shot noise. Other models show that an enhanced shot noise can be explained by coupling effects either to a nearby localized state (Ref. [4]) or by coupling between electrons and phonons (Ref. [11]). Ref. [12] explains a super-Poissonian shot noise and a double peak in the Fano factor in resonant tunneling through two vertically coupled QDs by Coulomb interaction of single-particle and doubly-occupied states at the same bias voltage. Another theoretical model describes transport through four metallic dots arranged as two parallel, only capacitively coupled stacks of two dots and shows not only an enhanced shot noise but also a double-peak behavior in the Fano factor [13]. The enhancement in this model depends on the ratio between the tunneling resistances of each parallel pair of QDs. This could be the reason for the strong temperature dependence of the shot noise power [14].

In conclusion, we have shown a reproducible doublepeak behavior in the Fano factor $\alpha$ with values above 1 , i.e. super-Poissonian shot noise, in the range of a current peak on coupled self-assembled QDs.

The authors would like to thank the BMBF for financial support.

\section{REFERENCES}

1. W. Schottky, Annalen der Physik (Leipzig) 57, 541 (1918).

2. H. Birk, M. J. M. de Jong, and C. Schönenberger, Phys. Rev. Lett. 75, 1610 (1995).

3. A. Nauen, I. Hapke-Wurst, F. Hohls, U. Zeitler, R. J. Haug, and K. Pierz, Phys. Rev. B 66, 161303 (2002).

4. S. S. Safanov, A. K. Savchenko, D. A. Bagrets, O. N. Jouravlev, Y. V. Nazarov, E. H. Linfield, and D. A. Ritchie, Phys. Rev. Lett. 91, 136801 (2003).

5. A. Nauen, F. Hohls, N. Maire, K. Pierz, and R. J. Haug, Phys. Rev. B 70, 033305 (2004).

6. D. Sarkar, U. Zeitler, I. Hapke-Wurst, R. J. Haug, and K. Pierz, Proc. 26th Int. Conf. Phys. of Semiconductors, Edinburgh, IOP Conference Series 171, (IOP, Bristol, 2003), P233 (2002).

7. I. Hapke-Wurst, U. Zeitler, U. F. Keyser, R. J. Haug, K. Pierz, and Z. Ma, Appl. Phys. Lett. 82, 1209 (2003).

8. H. Eisele, O. Flebbe, T. Kalka, C. Preinesberger, F. Heinrichsdorff, A. Krost, D. Bimberg, and M. DähnePietsch, Appl. Phys. Lett. 75, 106 (1999).

9. T. Bryllert, M. Borgstrom, L.-E. Wernersson, W. Seifert, and L. Samuelson, Appl. Phys. Lett. 82, 2655 (2003).

10. G. Kießlich, P. Samuelsson, A. Wacker, and E. Schöll, Phys. Rev. B 73, 033312 (2006).

11. J. Koch, and F. von Oppen, Phys. Rev. Lett. 94, 206804 (2005).

12. G. Kießlich, A. Wacker, and E. Schöll, Nonequilibrium Carrier Dynamics in Semiconductors Proc. 14th Int. Conf., July, 2005, Chicago, USA Series: Springer Proc. Phys., Vol. 110 (2006).

13. M. Gattobigio, G. Iannaccone, and M. Macucci, Phys. Rev. B 65, 115337 (2002).

14. P. Barthold, F. Hohls, N. Maire, K. Pierz, and R. J. Haug, Phys. Rev. Lett. 96, 246804 (2006). 